\title{
TITLE:
}

\section{A Temperature Shift Method in Canonical Molecular Dynamics}

$\operatorname{AUTHOR}(\mathrm{S})$ :

OTSUKA, Hiromi

\section{CITATION:}

OTSUKA, Hiromi. A Temperature Shift Method in Canonical Molecular Dynamics. 物性研 究 1989, 52(3): 276-298

ISSUE DATE:

1989-06-20

URL:

http://hdl.handle.net/2433/93619

RIGHT: 
カノニカル分子動力学における温度 shift の方法

\section{大阪大学 理学部 大塚博巳}

カノニカル分子動力学法の 1 つである能勢の方法では、興味の対照となって いる物理系の自由度に加え、その系とエネルギーをやり取りする”熱浴”の自由 度 $\left(s, P_{s}\right)$ が、導入される。この熱浴変数 $s$ のべき乗 $s^{k}$ は、sampling を行な う trajectory 上において物理系に対し、 $\exp (-\beta(k) H) / \exp (-\beta H)$ と、同等の性 質を持っている。このことから $s^{k}$ を weight として平均をとることにより、実

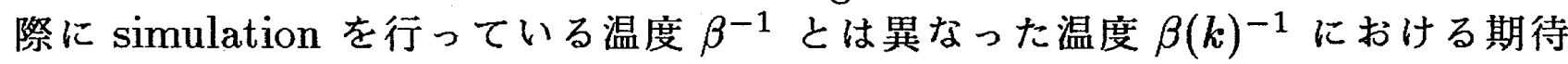
值を得ることができる。更に、べき係数 $k$ を無限小だけ変化させることにより、 この shift した温度 $\beta(k)^{-1}$ における温度微分量を与える関係式が得られる。 定温定圧分子動力学法の場合に以上の関係式を拡張する事る容易である。

これらの方法の有効性を確かめるために、我々は 13-atom Lennerd-Jones cluster に関する simulation を行った。

\section{Contents}

$\S 1$ Introduction

$\S 2$ Formulation

2.1 Nosé's method

2.2 Formulation of Temperature Shift Method

$\S 3$ T-const.MD Simulation on a 13-atom Lennerd-Jones Cluster

$\S 4$ Conclusions

Appendix A

Appendix B

References 


\section{A Temperature Shift Method in Canonical Molecular Dynamics}

\section{Hiromi OTSUKA}

We propose a method for obtaining the average values of physical quantities at temperatures different from the assumed value from a simulation calculation of the constant temperature molecular dynamics (MD) with a fixed temperature. The method is developed on the basis of Nosé's canonical MD. The present method is numerically tested in a simulation on a 13-atom Lennard-Jones cluster.

\section{$\S 1$ Introduction}

Recently, several methods of molecular dynamics (MD) for treating ensembles other than the microcanonical ensemble have been developed; they are the constant temperature ( $T$-const.) MD methods, the constant temperature constant pressure (PT-const.) MD methods, and others. ${ }^{1-4)}$ Among them, the method proposed by Nose $e^{3,4)}$ has attracted much attention, being simple and transparent. He introduced an extra degree of freedom described by a coordinate and its canonical conjugate momentum to represent a "heat bath" which exchanges energy with a given physical system. By choosing an appropriate potential for the heat bath, Nosé showed that the microcanonical ensemble for the extended system consisting of the heat bath and the physical system is equivalent to the canonical ensemble of the physical system for a constant temperature. He also showed that the T-const. MD method developed on the basis of this device could be extended to the PT-const. MD by combining it with Andersen's constant pressure MD method ${ }^{1)}$; in this case the volume of the physical system 
大榢 博已

is treated as a dynamical variable. Hoover ${ }^{5}$ ) gave a further elucidation of Nosés method by showing that the heat bath variables act as thermodynamic friction.

An interesting method for calculating the free energy by means of Nosé's method was developed by Brańka and Parrinello. ${ }^{6)}$ They showed that the calculation of the average of the $k$-th power of the heat bath variable $s$, i.e., $s^{k}$, by T-const. MD at a temperature $\beta^{-1}$ yields the free energy difference between $\beta^{-1}$ and another temperature $\beta(k)^{-1}$; the latter temperature $\beta(k)^{-1}$ is determined by $\beta^{-1}$ and $k$.

In this paper we extend the above-mentioned Brańka and Parrinello method to show that the average of a given physical quantity $A$ at the temperature $\beta(k)^{-1}$ can be obtained by taking a ratio of $\left\langle s^{k} A>_{\beta}\right.$ to $\left\langle s^{k}>_{\beta}\right.$, where $<$ $\ldots>_{\beta}$ denotes the average value calculated by $\mathrm{T}$-const. $\mathrm{MD}$ at the temperature $\beta^{-1}$. This result can be extended to the PT-const. MD. By varying the power parameter $k$, we can obtain information at various temperatures $\beta(k)^{-1}$ by a simulation calculation at $\beta^{-1}$; we can also obtain a temperature derivative of the average of a physical quantity by adapting the present method.

This paper consists of four sections. The basic formulation is given in $\S 2$. In $\S 3$, we perform a T-const. MD simulation on a 13-atom Lennard-Jones cluster to check the validity of the method. A summary and conclusions are given in $\S 4$. 


\section{$\S 2$ Formulation}

\subsection{Nosé's method}

In this subsection, we shall summarize Nosé's method briefly. ${ }^{3,4)}$ Nosé's Hamiltonian for T-const. MD is given by

$$
H_{n}\left(q, p, s, P_{s}\right)=H\left(q, \frac{p}{s}\right)+\frac{P_{s}^{2}}{2 Q}+\frac{f+1}{\beta} \ln s,
$$

where $H$ is the Hamiltonian for a physical system and $\beta^{-1}$ is the simulation temperature of the system. $f$ denotes the degrees of freedom of the physical system: $f=3 N$ for the $N$-particle system in three dimensions. When the system has conserved quantities, this equation must be corrected. $Q, s$, and $P_{s}$ are the mass, the coordinate, and its conjugate momentum for the motion of the heat bath, respectively. $(q, p, d t)$ are the virtual variables introduced by Nosé; they are related to the real variables $\left(q^{\prime}, p^{\prime}, d t^{\prime}\right)$ representing coordinates, momenta and the time of the physical system by

$$
\left(q, \frac{p}{s}, \frac{d t}{s}\right)=\left(q^{\prime}, p^{\prime}, d t^{\prime}\right)
$$

We assume that these virtual variables obey Hamilton's equations of motion with Hamiltonian eq.(2.1). We write their trajectory as $\left(q(t), p(t), s(t), P_{s}(t)\right)$. With the quasi-ergodic hypothesis, the long-time average of a certain physical quantity $A(q, p / s)$ along this trajectory is equivalent to the average which is calculated by a uniform sampling from the equienergy surface in the extended phase space $\left(q, p, s, P_{s}\right)$ :

$$
\begin{aligned}
\lim _{t \rightarrow \infty} \frac{1}{t} \int d t A\left(q(t), \frac{p(t)}{s(t)}\right) & =\frac{\int d \Gamma A(q, p / s) \delta\left(E_{0}-H_{n}\right)}{\int d \Gamma \delta\left(E_{0}-H_{n}\right)} \\
& \equiv<A>_{\beta}
\end{aligned}
$$


where $d \Gamma=d q d p d s d P_{s}$ and $E_{0}$ is a constant which is determined by the initial condition of the extended system. We transform the virtual variables $p, q$ to the real variables $p^{\prime}, q^{\prime}$ by use of eq.(2.2), and using the equivalence relation for $\delta$-function:

$$
\delta(g(x))=\delta\left(x-x_{0}\right) / \frac{d g(x)}{d x}
$$

where $x_{0}$ is the zero of $g(x)$, we can rewrite eq.(2.3) as

$$
<A>_{\beta}=\frac{\int d \Gamma^{\prime} d s d P_{s} A\left(q^{\prime}, p^{\prime}\right) s^{f+1} \delta\left(s-s_{0}\right)}{\int d \Gamma^{\prime} d s d P_{s} s^{f+1} \delta\left(s-s_{0}\right)}
$$

where $d \Gamma^{\prime}=d q^{\prime} d p^{\prime}$ and

$$
s_{0}=\exp \left[\frac{\beta}{f+1}\left\{E_{0}-\frac{P_{s}^{2}}{2 Q}-H\left(q^{\prime}, p^{\prime}\right)\right\}\right]
$$

By integrating with respect to the heat bath variables $s$ and $P_{s}$, we can derive following equation.

$$
\begin{aligned}
<A>_{\beta} & =\frac{\int d \Gamma^{\prime} A\left(q^{\prime}, p^{\prime}\right) e^{-\beta H\left(q^{\prime}, p^{\prime}\right)}}{\int d \Gamma^{\prime} e^{-\beta H\left(q^{\prime}, p^{\prime}\right)}} \\
& \equiv<A>_{\beta}^{C},
\end{aligned}
$$

where $\langle\ldots\rangle_{\beta}^{C}$ denotes the average with the canonical ensemble at $\beta^{-1}$.

In the case of PT-const. MD, Nosé's Hamiltonian corresponding to eq.(2.1) is

$$
H_{n}\left(q, p, s, P_{s}, V, P_{v}\right)=H\left(q V^{1 / 3}, \frac{p}{s V^{1 / 3}}\right)+\frac{P_{s}^{2}}{2 Q}+\frac{f+1}{\beta} \ln s+\frac{P_{v}^{2}}{2 W}+P_{e x} V
$$


where $P_{e x}$ denotes the simulation pressure. $W, V$, and $P_{v}$ are the mass, the coordinate, and the momentum for the motion of the volume variable, respectively. The relations between the real variables and the virtual variables are given by

$$
\left(q V^{1 / 3}, \frac{p}{s V^{1 / 3}}, \frac{d t}{\dot{s}}\right)=\left(q^{\prime}, p^{\prime}, d t^{\prime}\right) .
$$

The following expression is derived in the same way as the case of T-const. MD.

$$
\begin{aligned}
& \lim _{t \rightarrow \infty} \frac{1}{t} \int d t A\left(q(t) V(t)^{1 / 3}, \frac{p(t)}{s(t) V(t)^{1 / 3}}\right) \\
= & \frac{\int d \Gamma d V d P_{v} A\left(q V^{1 / 3}, p / s V^{1 / 3}\right) \delta\left(E_{0}-H_{n}\right)}{\int d \Gamma d V d P_{v} \delta\left(E_{0}-H_{n}\right)} \equiv<A>_{\beta}, P_{e x} \\
= & \frac{\int d V d \Gamma^{\prime} A\left(q^{\prime}, p^{\prime}\right) e^{-\beta\left(H\left(q^{\prime}, p^{\prime}\right)+P_{e x} V\right)}}{\int d V d \Gamma^{\prime} e^{-\beta\left(H\left(q^{\prime}, p^{\prime}\right)+P_{e x} V\right)}} \\
= & <A>_{\beta, P_{e x}}^{C} .
\end{aligned}
$$

Consequently, by choosing Ins to the potential for heat bath variables and generating microcanonical ensembles for extended systems, we can take canonical ensembles for physical systems.

The above argument applies to the case of the virtual time sampling, i.e., the sampling along the trajectory $(q(t), p(t), s(t))$. On the other hand, in the case of real time sampling, which is performed along $\left(q^{\prime}\left(t^{\prime}\right), p^{\prime}\left(t^{\prime}\right)\right)$, Nosé pointed out that the factor $f+1$ must be replaced by $f$. For example, Nosé's Hamiltonian eq.(2.1) becomes

$$
H_{n}\left(q, p, s, P_{s}\right)=H\left(q, \frac{p}{s}\right)+\frac{P_{s}^{2}}{2 Q}+\frac{f}{\beta} \ln s .
$$

We perform a simulation on a 13-atom Lennerd-Jones cluster in $\S 3$ using the real variables; the factor $f+1$ in all equations is replaced by $f$. The detailed 
大塚 博巳

explanation of the relation between the real time sampling and the virtual time sampling is given in Appendix A.

\subsection{Formulation of temperature shift method}

We have explained an outline of Nosé's method in the above subsection. Next, let us consider the following quantity to formulate "temperature shift method".

$$
\frac{\left\langle s^{k} A>_{\beta}\right.}{<s^{k}>_{\beta}}=\frac{\lim _{t \rightarrow \infty} \frac{1}{t} \int d t s(t)^{k} A\left(q(t), \frac{p(t)}{s(t)}\right)}{\lim _{t \rightarrow \infty} \frac{1}{t} \int d t s(t)^{k}} .
$$

The right-hand side of this equation is rewritten in the same way as the derivation of eq.(2.5). Assuming that we calculate both the numerator and the denominator on the same equienergy surface, we obtain the next expression:

$$
\begin{aligned}
\frac{<s^{k} A>_{\beta}}{<s^{k}>_{\beta}} & =\frac{\int d \Gamma^{\prime} A\left(q^{\prime}, p^{\prime}\right) e^{-\beta(k) H\left(q^{\prime}, p^{\prime}\right)} / \int d \Gamma^{\prime} e^{-\beta H\left(q^{\prime}, p^{\prime}\right)}}{\int d \Gamma^{\prime} e^{-\beta(k) H\left(q^{\prime}, p^{\prime}\right)} / \int d \Gamma^{\prime} e^{-\beta H\left(q^{\prime}, p^{\prime}\right)}} \\
& =<A>_{\beta(k)}^{C},
\end{aligned}
$$

where

$$
\beta(k) \equiv\left(1+\frac{k}{f+1}\right) \beta
$$

Therefore, we can say from this equation that by calculating $\left\langle s^{k} A>_{\beta} /<\right.$ $s^{k}>_{\beta}$ by the T-const. $M D$ at $\beta^{-1}$, we obtain the average of $A$ at $\beta(k)^{-1}$.

By differentiating both sides of eq.(2.10) with respect to $k$, we derive the following expression for the temperature derivative:

$$
\frac{d<A>_{\beta(k)}^{C}}{d \beta(k)}=\frac{f+1}{\beta} \frac{<\left(s^{k} \ln s\right) A>_{\beta}<s^{k}>_{\beta}-<s^{k} A>_{\beta}<s^{k} \ln s>_{\beta}}{<s^{k}>_{\beta}^{2},}
$$


which can be rewritten as

$$
<\left(\frac{f+1}{\beta} \ln s-<\frac{f+1}{\beta} \ln s>_{\beta(k)}\right)\left(A-<A>_{\beta(k)}\right)>_{\beta(k)}
$$

The expression (2.13) can also be derived by taking into account the fact that the fluctuation of the total energy of the physical system is equivalent to that of the heat bath except for its sign. Using this equation we can calculate the temperature derivative at $\beta(k)^{-1}$ by the T-const. MD simulation at $\beta^{-1}$. For example, if $A$ equals the total energy of the physical system then the right-hand sides of eqs.(2.12) and (2.13) become

$$
-<\left(H-<H>_{\beta(k)}^{C}\right)^{2}>_{\beta(k)}^{C}
$$

This expression is familiar to us (see Appendix B).

It is a straightforward operation to extend the above argument to the case of PT-const. MD. We can easily derive expressions corresponding to eqs.(2.10) and (2.13) by paying attention to table I. The result is the following:

$$
\begin{gathered}
<A>_{\beta(k), P_{e x}(h)}^{C}=\frac{<e^{-\beta(k) V h} s^{k} A>_{\beta, P_{e x}}}{<e^{-\beta(k) V h} s^{k}>_{\beta, P_{e x}}} \\
\begin{aligned}
\frac{d<A>_{\beta(k), P_{e x}(h)}^{C}=}{d \beta(k)} & =\left(\frac{f+1}{\beta} \ln s+h V-<\frac{f+1}{\beta} \ln s+h V>_{\beta(k), P_{e x}(h)}\right) \\
& \left(A-<A>_{\beta(k), P_{e x}(h)}\right)>_{\beta(k), P_{e x}(h)} \\
\frac{d<A>_{\beta(k), P_{e x}(h)}^{C}=\beta(k)<\left(V-<V>_{\beta(k), P_{e x}(h)}\right)}{d P_{e x}(h)} & \left(A-<A>_{\beta(k), P_{e x}(h)}\right)>_{\beta(k), P_{e x}(h)}
\end{aligned} \\
\quad-283-
\end{gathered}
$$


大塚 博巳

where

$$
P_{e x}(h) \equiv P_{e x}+h
$$

We have obtained all the equations to be derived; we can make the meaning of these equations clearer by studying the behavior of $s$ on the trajectory. The total energy of the extended system $E_{0}$ is the conserved quantity of the motion:

$$
E_{0}=H\left(q, \frac{p}{s}\right)+\frac{P_{s}^{2}}{2 Q}+\frac{f+1}{\beta} \ln s .
$$

From this equation,

$$
\begin{aligned}
s^{k} & =\exp \left[\frac{k \beta}{f+1}\left\{E_{0}-\frac{P_{s}^{2}}{2 Q}-H\left(q^{\prime}, p^{\prime}\right)\right\}\right] \\
& =\text { const. } \exp \left[-(\beta(k)-\beta) P_{s}^{2} / 2 Q\right] \frac{\exp (-\beta(k) H)}{\exp (-\beta H)} .
\end{aligned}
$$

As we explained in Appendix $\mathrm{B}, \exp \left[-(\beta(k)-\beta) P_{s}^{2} / 2 Q\right]$ does not influence the averages of the physical quantities. Consequently, $s^{k}$ essentially has the same function as $e^{-\beta(k) H} / e^{-\beta H}$ and can shift the temperature from $\beta^{-1}$ to $\beta^{-1}(k)$. It is the same with PT-const. MD. We can show that $e^{-\beta(k) V h} s^{k}$ is essentially equal to $e^{-\beta(k)\left(H+P_{e x}(h) V\right)} / e^{-\beta\left(H+P_{e x} V\right)}$; it can shift both the temperature and the pressure from $\beta^{-1}, P_{e x}$ to $\beta(k)^{-1}, P_{e x}(h)$, respectively.

Table I The relation between the weights at which temperature and pressure shifts occur.

\begin{tabular}{l|ll} 
& Temperature & Pressure \\
\hline Potential & $\frac{f+1}{\beta} \ln s$ & $P_{e x} V$ \\
Weight & $s^{k}$ & $\left(e^{-\beta V}\right)^{h}$
\end{tabular}




\section{$\S 3$ T-const. MD Simulation on a 13-atom Lennard-Jones Cluster}

Our simulations were carried out on a system of 13 atoms interacting with a Lennard-Jones (LJ) potential:

$$
U_{i j}=4 \epsilon\left(\left(\frac{\sigma}{R_{i j}}\right)^{12}-\left(\frac{\sigma}{R_{i j}}\right)^{6}\right)
$$

It is well known that the LJ potential is a good approximation for rare gases such as argon, in which case $\epsilon=125 k_{B}, \sigma=3.45 A$. We measure energy in units of $\epsilon$, length in $\sigma$, temperature in $\left(\sigma / k_{B}\right)$ and time in $\left(m \sigma^{2} / \epsilon\right)^{1 / 2}$, where $m$ denotes the mass of the atom. The physical system was enclosed in a $6 \times 6 \times 6$ box.

We used the real variables. This is because the behavior of the real variables is more moderate than that of the virtual variables. Therefore, the real variables are more suitable for the simulation than the virtual variables. The integration of the differential equation was performed on the NEC SX-2N at Computation Center of Osaka University using a fourth-order Runge-Kutta method in double precision. We rewrite eq.(2. 1') using the real variables:

$$
H_{n}^{\prime}=H\left(q^{\prime}, p^{\prime}\right)+\frac{\left(s^{\prime} P_{s}^{\prime}\right)^{2}}{2 Q}+\frac{f}{\beta} \ln s^{\prime}
$$

where $s^{\prime}=s, P_{s}^{\prime}=P_{s} / s$. This quantity is no longer the Hamiltonian for the motion of the real variables, but it is still conserved. Therefore, we can take an appropriate time step for integration by paying attention to its behavior.

We performed seven different simulations at the following temperatures: $\beta^{-1}=0.34303,0.4288,0.536,0.67,0.8375,1.1167$, and 1.488 . After appropriate equilibration run, the averages were taken over a run of $1,000,000$ steps at each temperature. At each simulation we calculated the averages for $k=10,9$, $\ldots,-10$. 
First, we show configurations of the 13 -atom cluster at the above temperatures in fig.1. Frames in this figure show the $6 \times 6 \times 6$ box. From these configurations, we can recognize some differences between the configurations at $\beta^{-1}=0.34074,0.47889$ and others. It appears that the cluster at these two temperatures is in the state which is different from at other temperatures. Therefore, we treat results at $\beta^{-1}=0.34043$ and 0.47889 in the end of this section.

We calculated four physical quantities: the kinetic energy, the total energy, the mean bond length (MBL), and the specific heat of the physical system. Errors for these data were also estimated from the standard deviations of the short-time averages, which were calculated from 20,000 steps simulations. We shall unify in the following figures that open squares, filled triangles, open circles, filled squares, and open triangles correspond to the simulations at $\beta^{-1}=1.488$, $1.1167,0.8375,0.67$, and 0.536 , respectively.

Figure 2 compares $\beta(k)^{-1}$ with the calculated temperatures from kinetic energy defined by

$$
T(\beta, k)=\frac{2}{f} \frac{<s^{\prime k} \sum_{i} \frac{\left(p_{i}^{\prime}\right)^{2}}{2}>_{\beta}}{<s^{\prime k}>_{\beta}}
$$

which are shown by above-mentioned symbols with error bars. The abscissa shows the value of $k$. These two temperatures for $|k|<8$ coincide with each other within error bars. We may thus conclude that we have confirmed the temperature shift effect induced by $s^{k}$.

Figure(3.a) plots the total energy per atom given by

$$
E=\frac{<s^{\prime k} H\left(q^{\prime}, p^{\prime}\right)>_{\beta}}{N<s^{\prime k}>_{\beta}}
$$

against the calculated temperature. We plot the data for even $k$ 's at each $\beta^{-1}$. 
カノニカル分子動力学における温度 shift の方法
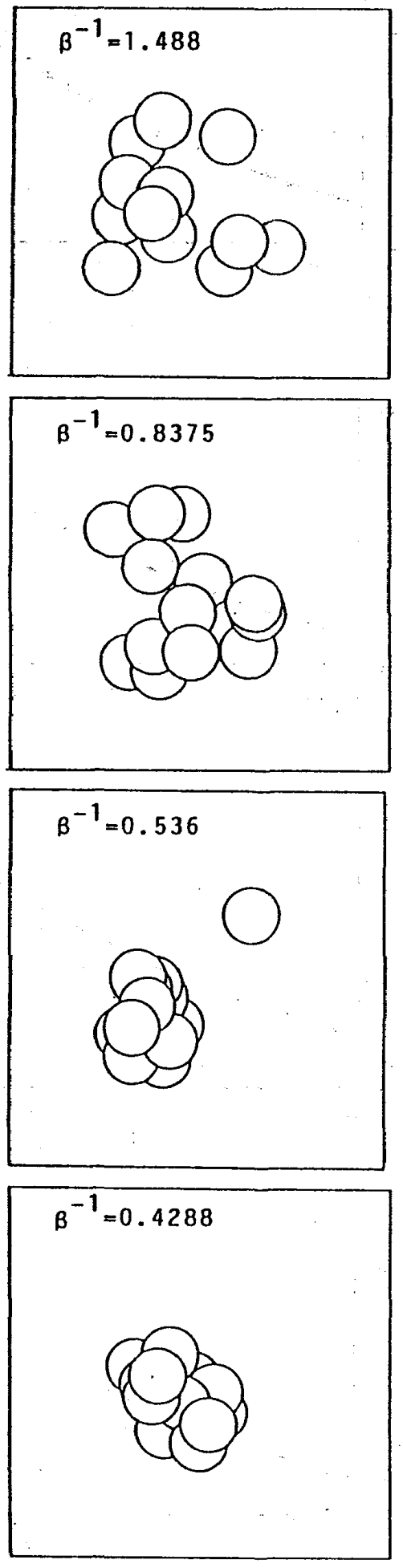
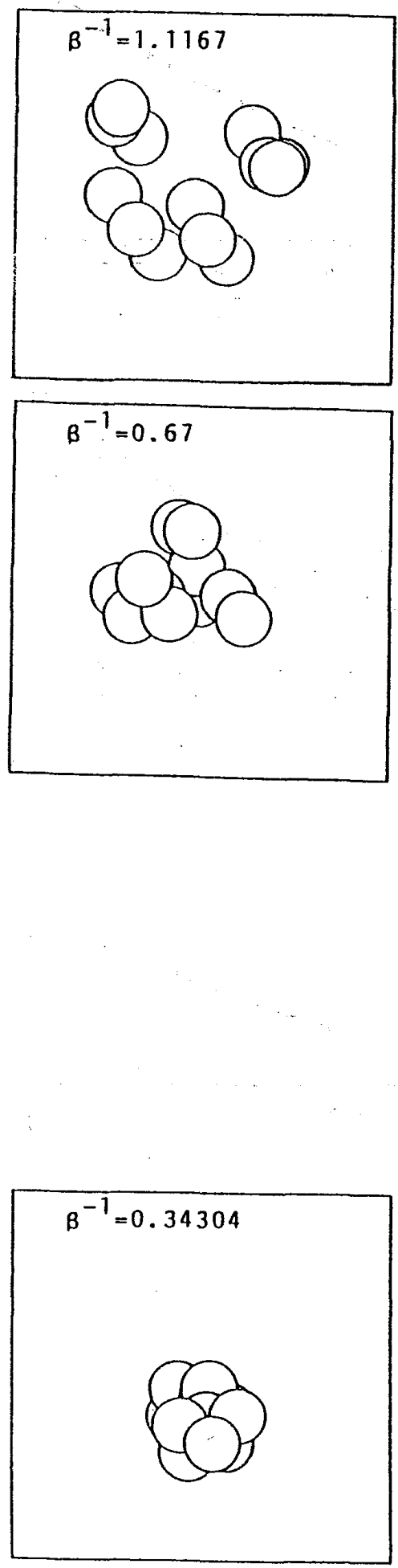

Fig.1 The configurations of the 13-atom $L J$ cluster at the simulation temperatures.

$-287-$ 

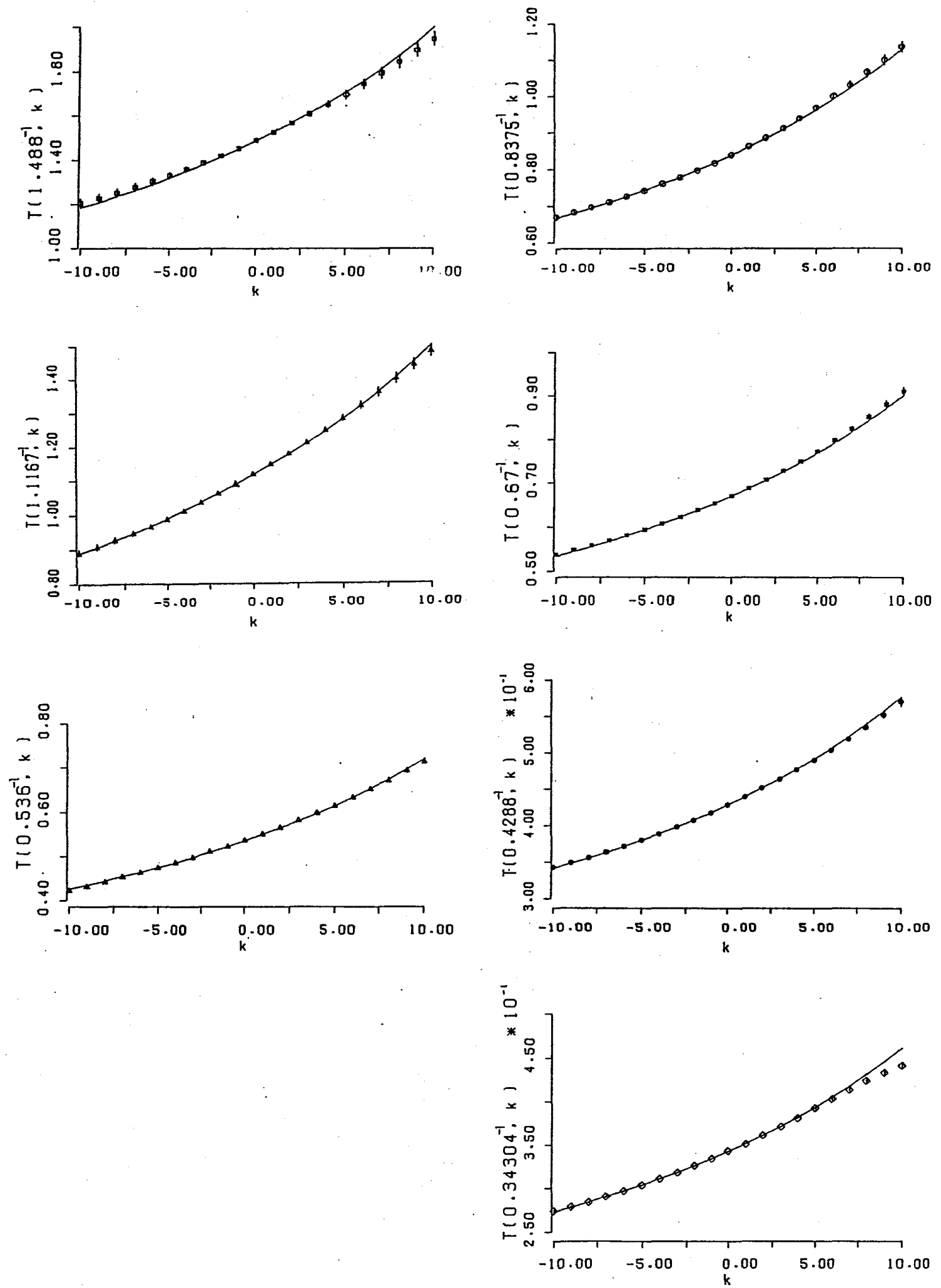

Fig.2 The relation between the calculated temperatures $T(\beta, k)$ which are denoted by symbols with error bars and the expected temperature curve $\beta(k)^{-1}$. 


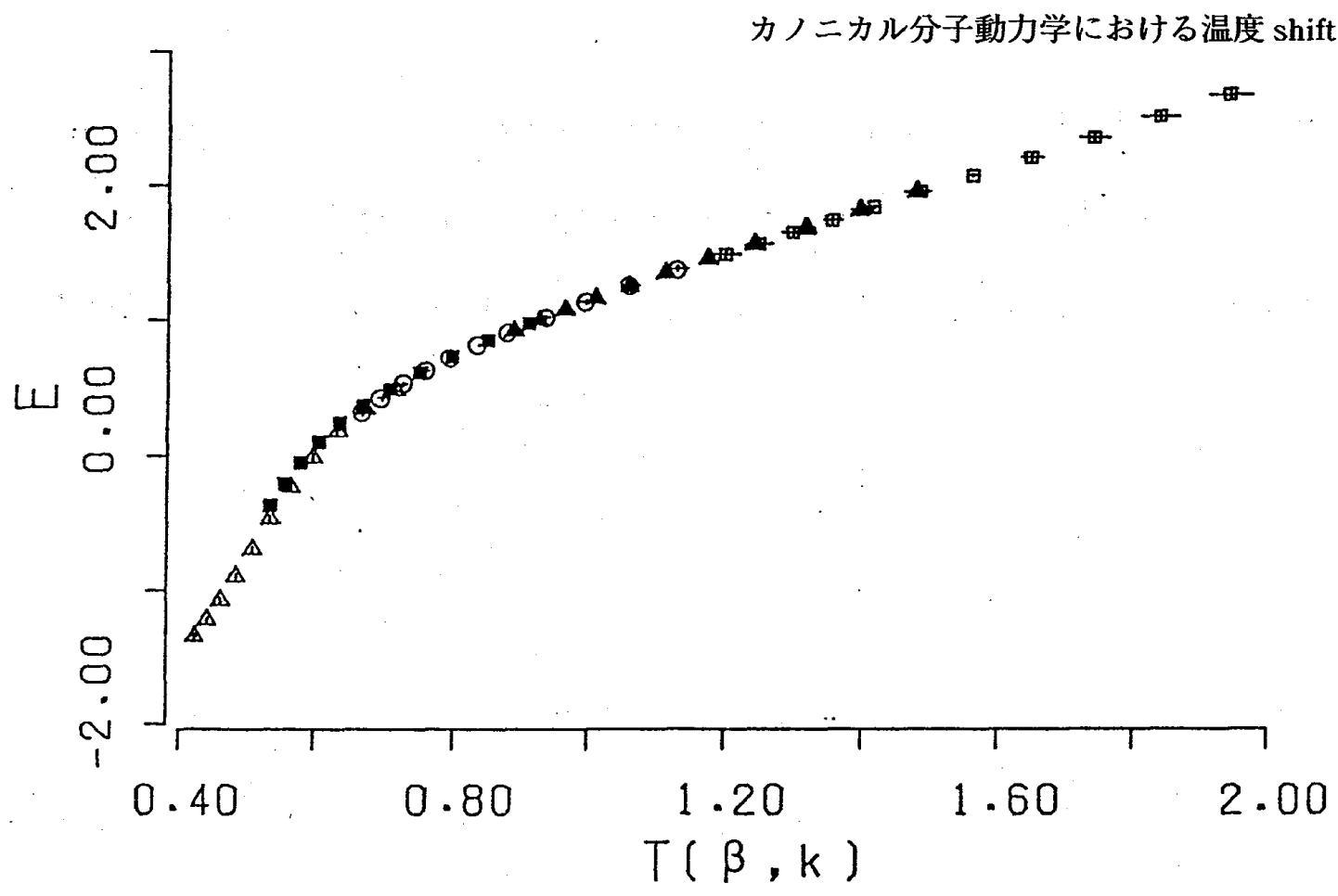

Fig.(3.a) The temperature dependence of the total energy per atom. The correspondence between the symbols and temperatures is the following: open triangles $\left(\beta^{-1}=0.536\right)$, filled squares $\left(\beta^{-1}=0.67\right)$, open circles $\left(\beta^{-1}=0.8375\right)$, filled triangles $\left(\beta^{-1}=1.1167\right)$, and open squares $\left(\beta^{-1}=1.4889\right)$.

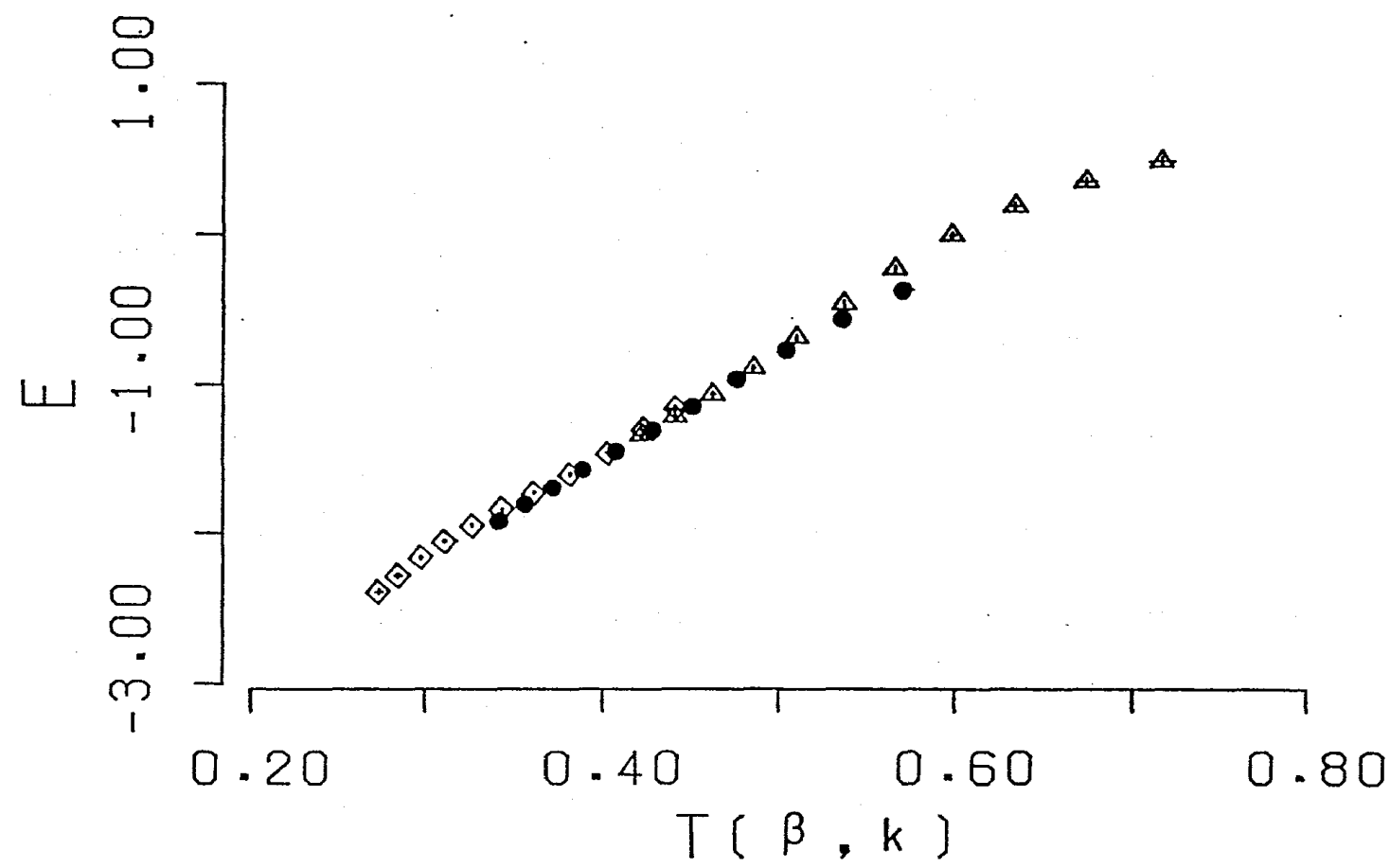

Fig.(3.b) The temperature dependence of the total energy per atom. The correspondence between the symbols and temperatures is the following: closed circles $\left(\beta^{-1}=0.4288\right)$ and open rhombus $\left(\beta^{-1}=0.34304\right)$. 


\section{大塚 博巳}

We can see from this figure that the data obtained from the simulations at five different temperatures are on a single smooth curve within error bars.

The last physical quantity to which eq.(2.10) is applied is the MBL:

$$
L_{B}=\frac{2<s^{\prime k} \sum_{i>j} R_{i j}^{\prime}>_{\beta}^{*}}{N(N-1)<s_{k}^{\prime}>_{\beta}}
$$

where $R_{i j}^{\prime}$ is the distance between the $\mathrm{i}$-th atom and the $\mathrm{j}$-th atom. We plot the results for MBL in fig.(4.a). As the temperature becomes higher, the $M B L$ comes to be saturated because of the presence of the boundary. We find that there is a temperature range near $T(\beta, k) \simeq 0.5$ where the $\mathrm{MBL}$ varies rapidly. The temperature shift method functions well even in this range.

We calculated the specific heat to check the expression for the temperature derivative eq.(2.13):

$$
C=-\frac{<\left(s^{\prime k} \frac{f}{\beta} \ln s^{\prime}\right) H>_{\beta}<s^{\prime k}>_{\beta}-<s^{\prime k} H>_{\beta}<s^{\prime k} \frac{f}{\beta} \ln s^{\prime}>_{\beta}}{N T(\beta, k)^{2}<s^{\prime k}>_{\beta}^{2}} .
$$

The results are shown in fig.(5.a). The values of specific heat obtained as derivatives $d E / d T(\beta, k)$ from fig.(3.a) are also given in this figure as five curves. Except for the data with large $|k|$ 's, these values are consistent with one another. This figure shows that there is a peak in a specific heat curve, and that it is in the above-mentioned temperature range, where the MBL changes rapidly. Therefore, we may consider this peak to correspond to the melting of the cluster. We can see from this figure that for a sufficiently high temperature, the specific heat per atom approaches $3 / 2$.

In order to study the influence of taking weighted averages on the distributions of physical quantities, we show histograms of the above calculated 
カノニカル分子動力学における温度 shift の方法



Fig.(4.a) The temperature dependence of the MBL. The correspondence between the symbols and the temperatures is the same as that in fig.(3.a).

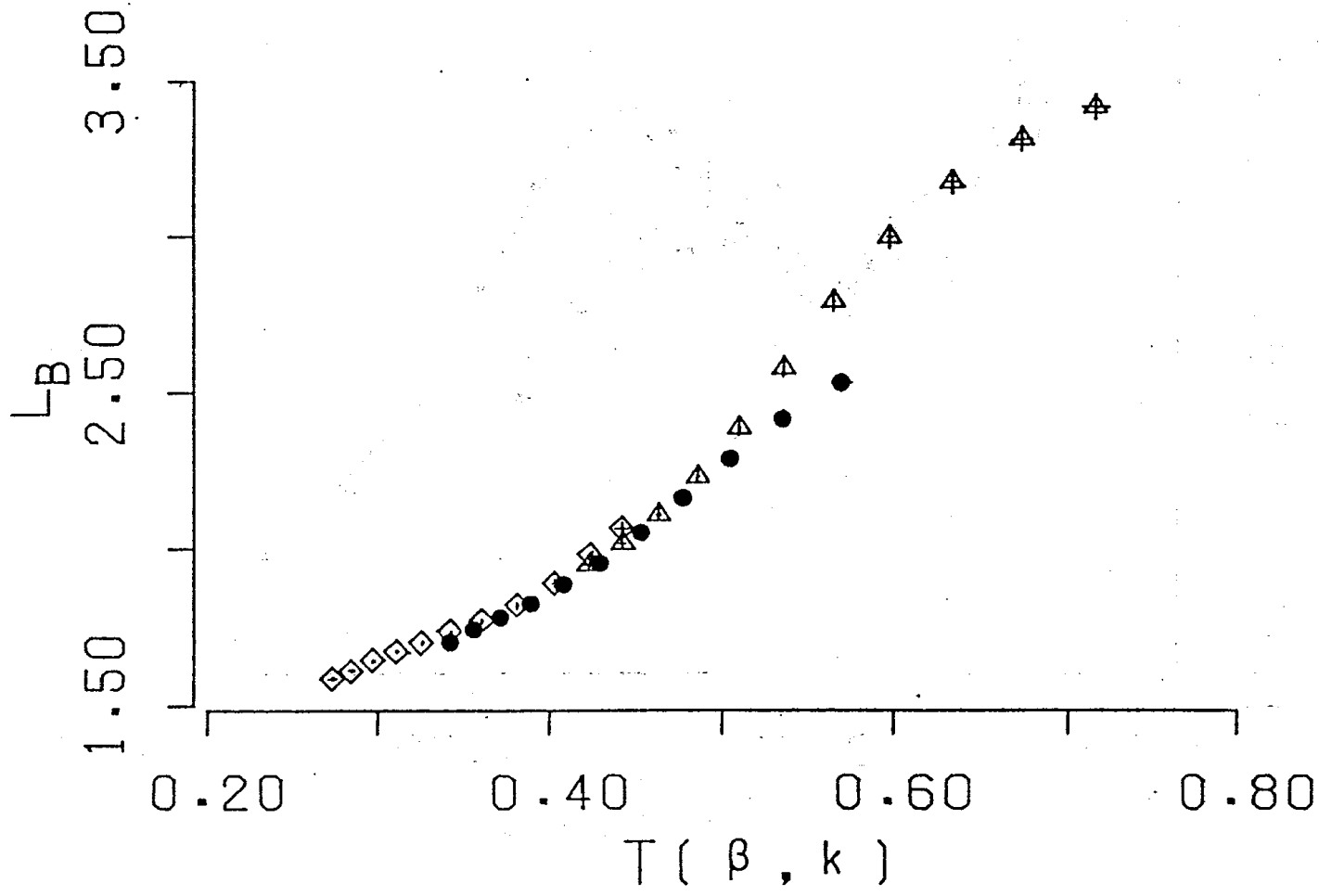

Fig.(4.b) The temperature dependence of the MBL. The correspondence between the symbols and the temperatures is the same as that in fig.(3.b) 
大塚 博巳

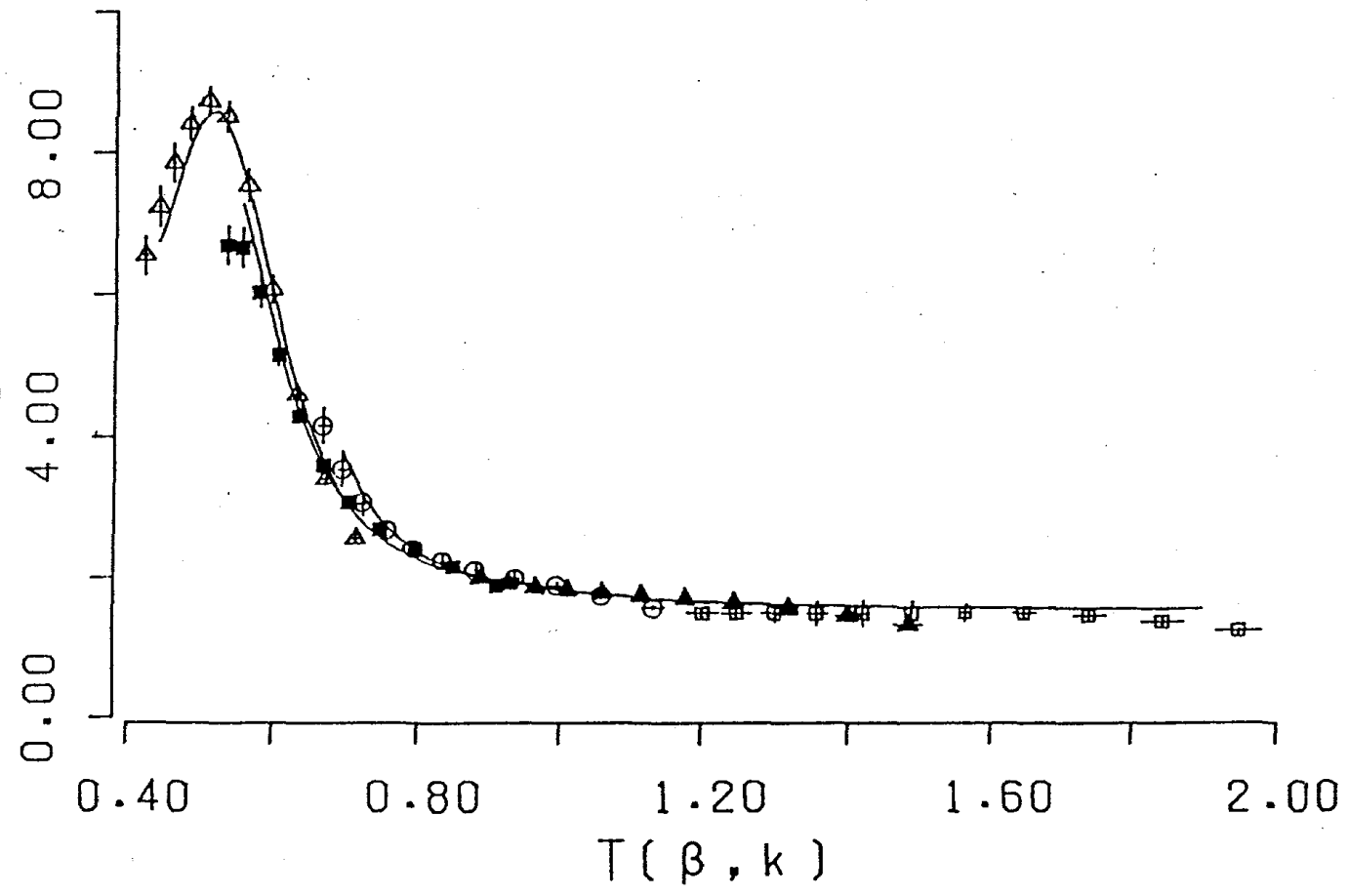

Fig.(5.a) The temperature dependence of the specific heat per atom. The correspondence between the symbols and the temperatures is the same as that in fig.(3.a). The symbols with error bars denote the values obtained using eq.(3.6) and the five curves show the specific heat caiculated from fig.(3.a) by $d E / T(\beta, k)$

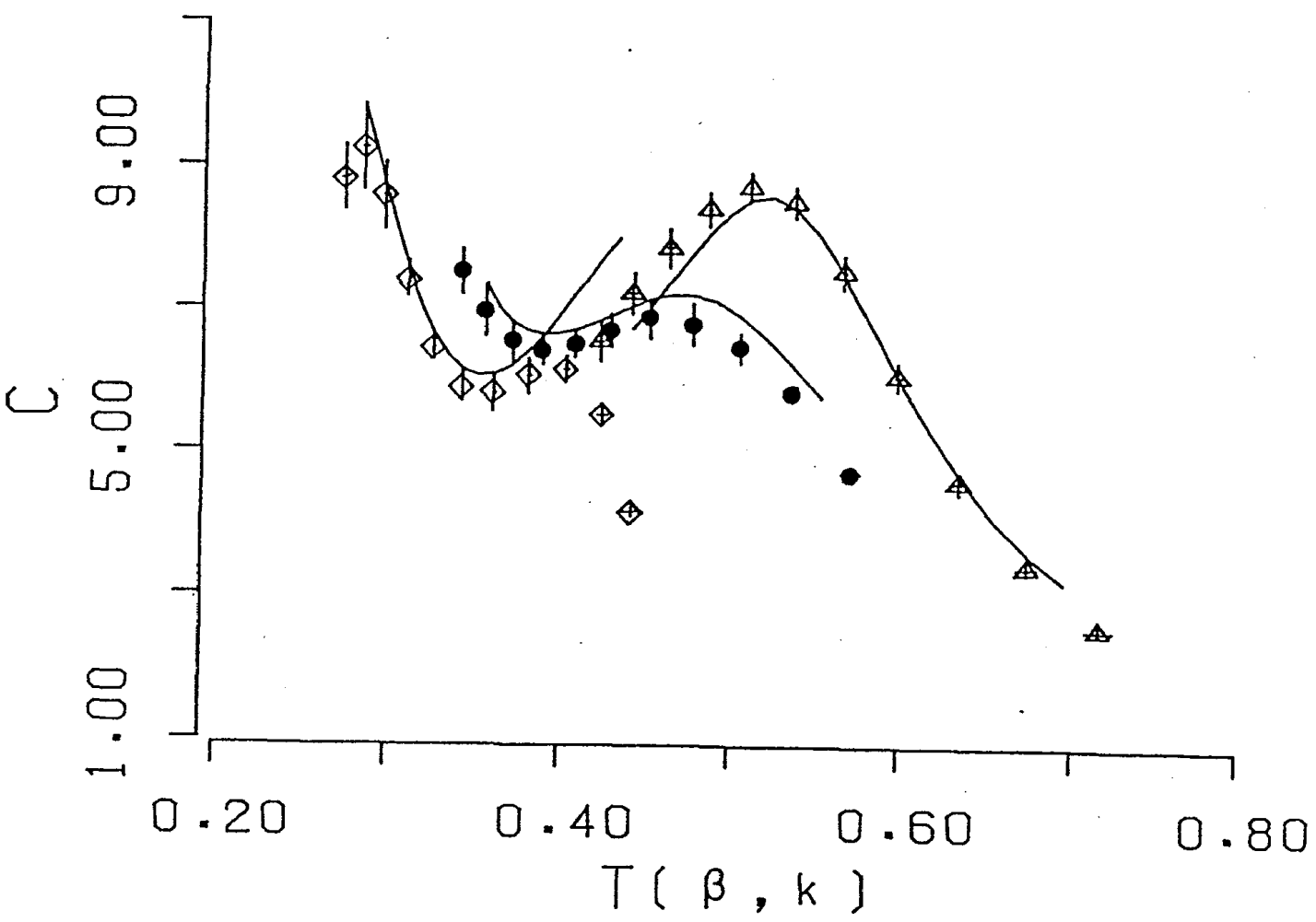

Fig.(5.b) The temperature dependence of the specific heat per atom. The correspondence between the symbols and the temperatures is the same as that in fig.(3.b). The symbols with error bars denote the values obtained using eq.(3.6) and the five curves show the specific heat calculated from fig.(3.b) by $d E / T(\beta, k)$ 
short-time averages of the kinetic energy (see fig.(6.a) fig.(6.e)). Nine figures correspond to distributions for $k=-4, \ldots 4$. In these figures, the long-time averages are also indicated by vertical lines, and the maximum and the minimum of short-time averages are additionally remarked. We can see from these figures how the weights $s^{k}$ shift the distributions of the kinetic energy.

From the above simulation calculation results, we could check the validity of eq.(2.10) and eq(2.13). However, it dose not always work well. Next, we show the results of $M D$ simulation at $\beta^{-1}=0.34303,0.4288$, and 0.534 . Figure 2, fig.(3.b), fig.(4.b), and fig.(5.b) plot the data of the calculated temperature, the total energy, the MBL, and the specific heat, respectively. The correspondence between simulation temperatures and the symbols is the following: the closed circles and open rhombus correspond to $\beta^{-1}=0.4288$ and 0.34304 . The consistency between data in fig.(3.b) and fig.(4.b) becomes worse than that in fig.(3.a) and fig.(4.a), still more, fig.(5.b) shows that eq.(2.13) does not work well at these temperatures. This would be because that the behavior of the cluster is not sufficienly chaotic to satisfy the quasi-ergodic hypothesis at these temperatures below the melting temperature. 

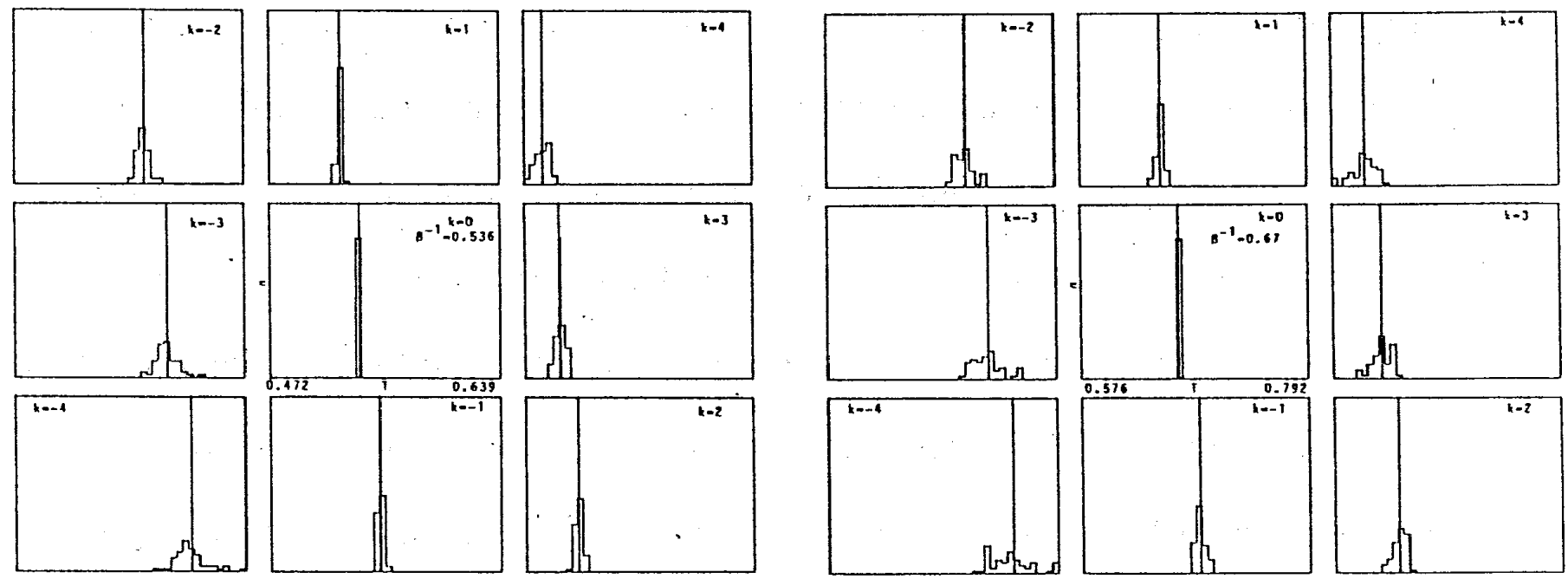

$F i_{B \cdot(6 . n)}$
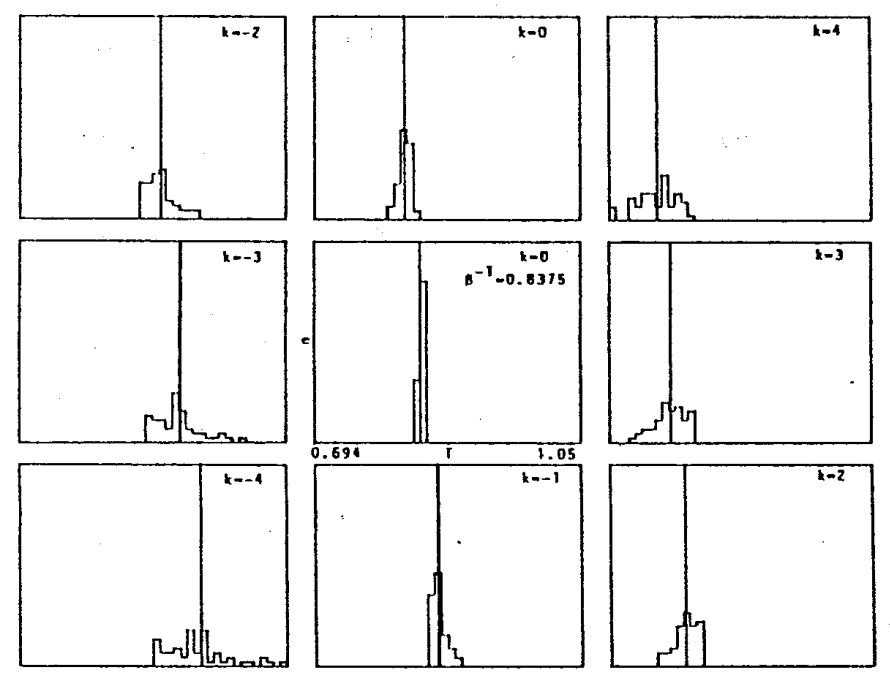

Fig.(B.c)
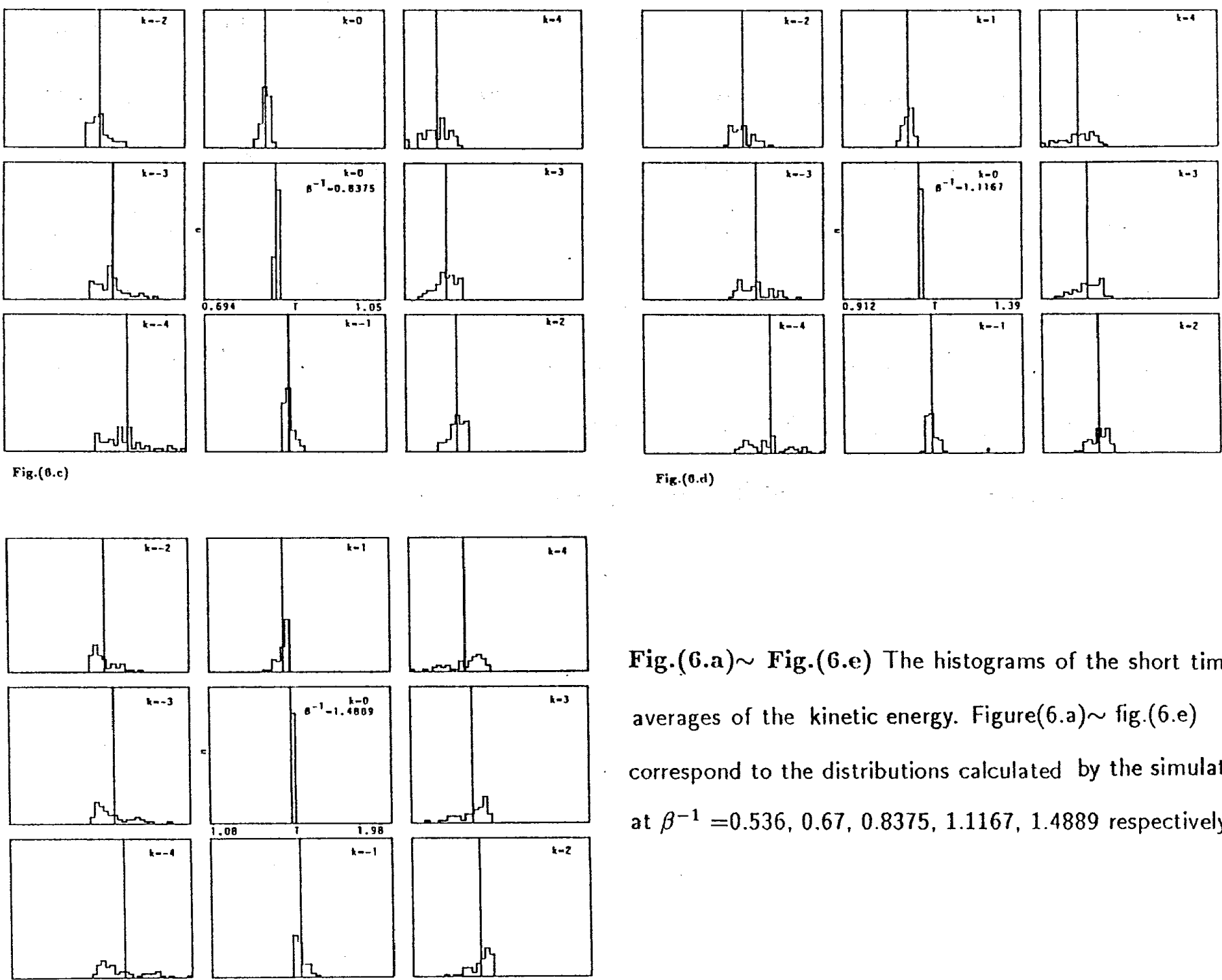

Lant



Fig.(6.a) Fig.(6.e) The histograms of the short time averages of the kinetic energy. Figure(6.a) fig.(6.e) correspond to the distributions calculated by the simulations at $\beta^{-1}=0.536,0.67,0.8375,1.1167,1.4889$ respectively.

Fig.(0.e) 


\section{$\S 4$ Conclusions}

We have shown that in the case of $\mathrm{T}$-const. MD, the weighted averages using $s^{k}$ give the averages of physical quantities at $\beta(k)^{-1}$, which is different from the simulation temperature $\beta^{-1}$, and that fluctuation of the function $\ln s$, which is the 0 -th power of $s$, yields the temperature derivatives instead of the temperature shift. It is easy to extend these relations to the case of PT-const MD: the weight which produces the pressure shift is $\left(e^{-\beta V}\right)^{h}$, and that which gives the pressure derivative is $\ln \left(e^{-\beta V h}\right)=-\beta h V$. We have checked the usefulness of these methods. Equation (2.10) shows that by calculating the weighted averages using $s^{k}$, we can obtain the temperature shift with a magnitude $k /(f+1+k) \beta$. Therefore, this method works well for a relatively small system; a 13-atom LJ cluster was chosen in $\S 3$. It is to be noted that at temperature much lower than the melting temperature, the present temperature shift method may not work well because of the breaking of quasi-ergodicity. When we take weighted averages using $s^{k}$ with large $|k|$, many steps are required to attain convergence. The method is useful because we can get information about various temperatures by one MD run at a certain temperature. We can say that these relations explored an unknown possibility of Nosé's method.

\section{Acknowledgment}

The author thanks Professor J. Kanamori, Dr. M. Kikuchi and Mr. Y. Sakamoto for helpful discussions. The present research was supported by a Grant-in-Aid for Scientific Research. 


\section{Appendix A}

We summarize equations of motion here. In Hamiltonian eq(2.1), we take $H=$ $p^{2} / 2 s^{2}+\phi(q)$. The equations which the virtual variables obey are

$$
\frac{d q}{d t}=\frac{p}{s^{2}}, \frac{d p}{d t}=-\frac{\partial \phi}{\partial q}, \frac{d s}{d t}=\frac{P_{s}}{Q}, \frac{d P_{s}}{d t}=\frac{1}{s}\left(\frac{p^{2}}{s^{2}}-\frac{f+1}{\beta}\right) .
$$

Using eq(2.2) and $s^{\prime}=s, P_{s}^{\prime}=P_{s} / s$, we can obtain equations for real variables:

$$
\frac{d q^{\prime}}{d t^{\prime}}=p^{\prime}, \frac{d p^{\prime}}{d t^{\prime}}=-\frac{\partial \phi}{\partial q^{\prime}}-p^{\prime} \zeta, \frac{d s^{\prime}}{d t^{\prime}}=s^{\prime} \zeta, \frac{d P_{s}^{\prime}}{d t^{\prime}}=\frac{1}{s^{\prime}}\left(p^{\prime 2}-\frac{g}{\beta}\right)-P_{s}^{\prime} \zeta
$$

where $\zeta=s^{\prime} P_{s}^{\prime} / Q$. We replaced $f+1$ by $g$ and it is determined later. As Hoover showed, if we take $\zeta$ as a new variable to describe the heat bath, the eq.(A.2) can be rewritten as

$$
\frac{d q^{\prime}}{d t^{\prime}}=p^{\prime}, \frac{d p^{\prime}}{d t^{\prime}}=-\frac{\partial \phi}{\partial q^{\prime}}-p^{\prime} \zeta, \frac{d \zeta}{d t^{\prime}}=\frac{1}{Q^{\prime}}\left(p^{2}-\frac{g}{\beta}\right)
$$

These are Vlasov type differential equations, in which the heat bath acts as a thermodynamic friction and have a canonical distribution as a stationary solution.

When we calculate the average of $A$ using the real time sampling,

$$
\begin{aligned}
& \lim _{t^{\prime} \rightarrow \infty} \frac{1}{t^{\prime}} \int d t^{\prime} A\left(q^{\prime}\left(t^{\prime}\right), p^{\prime}\left(t^{\prime}\right)\right) \\
= & \lim _{t \rightarrow \infty} \frac{1}{t} \int d t \frac{A(q(t), p(t) / s(t))}{s(t)} / \lim _{t \rightarrow \infty} \frac{1}{t} \int d t \frac{1}{s(t)} \\
= & \frac{\left\langle s^{-1} A>_{\beta^{\prime}}\right.}{<s^{-1}>_{\beta^{\prime}}} \\
= & <A>_{\beta^{\prime}(-1)}^{C},
\end{aligned}
$$

where $\beta^{\prime}(-1)=(1-1 /(f+1)) \beta^{\prime}$ from eq.(2.3) and $\beta^{\prime}=(f+1) \beta / g . g$ is determined by $\beta^{\prime}(-1)=\beta ; g$ is equal to $f$. Therefore, when we use the real variables, the factor $f+1$ must be replaced by $f$. 


\section{Appendix B}

The detailed derivation of eq.(2.14) is given in this appendix. For simplicity, we consider the case that $k=0$. From eq.(2.12),

$$
\begin{aligned}
& \frac{d<H(q, p / s)>_{\beta}^{C}}{d \beta}=\frac{f+1}{\beta}\left[<(\ln s) H(q, p / s)>_{\beta}-<H(q, p / s)>_{\beta}<\ln s>_{\beta}\right] \\
= & \frac{f+1}{\beta}\left[\frac{\int d \Gamma(\ln s) H \delta\left(E_{0}-H_{n}\right)}{\int d \Gamma \delta\left(E_{0}-H_{n}\right)}-\frac{\int d \Gamma(\ln s) \delta\left(E_{0}-H_{n}\right)}{\int d \Gamma \delta\left(E_{0}-H_{n}\right)} \frac{\int d \Gamma H \delta\left(E_{0}-H_{n}\right)}{\int d \Gamma \delta\left(E_{0}-H_{n}\right)}\right],
\end{aligned}
$$

where $d \Gamma=d q d p d s d P_{s}$. By transforming the virtual variables to the real variables and integrating with respect to $s$, we can rewrite the right-hand side of eq(B.1) as the following:

$$
\begin{aligned}
& -<\left(H-<H>_{\beta}^{C}\right)^{2}>_{\beta}^{C} \\
& -<\left(H\left(q^{\prime}, p^{\prime}\right)-<H\left(q^{\prime}, p^{\prime}\right)>_{\beta}^{C^{\prime}}\right)\left(\frac{P_{s}^{2}}{2 Q}-<\frac{P_{s}^{2}}{2 Q}>_{\beta}^{C^{\prime}}\right)>_{\beta}^{C^{\prime}} .
\end{aligned}
$$

The average $\langle\ldots\rangle_{\beta}^{C^{\prime}}$ is defined by

$$
<\alpha\left(q^{\prime}, p^{\prime}, P_{s}\right)>_{\beta}^{C^{\prime}}=\frac{\int d P_{s} d \Gamma^{\prime} \alpha\left(q^{\prime}, p^{\prime}, P_{s}\right) e^{-\beta\left(H\left(q^{\prime}, p^{\prime}\right)+\frac{P_{s}^{2}}{2 Q}\right)}}{\int d P_{s} d \Gamma^{\prime} e^{-\beta\left(H\left(q^{\prime}, p^{\prime}\right)+\frac{P_{s}^{2}}{2 Q}\right)}},
$$

where $d \Gamma^{\prime}=d q^{\prime} d p^{\prime} . H\left(q^{\prime}, p^{\prime}\right)$ dose not depend on $P_{s}$. Therefore, the fluctuation of total energy of the physical system does not correlate to that of the kinetic energy of the heat bath. Consequently, the second term of eq.(B.2) is equal to zero, and eq.(2.14) can be derived. This is not only in the case of the total energy, i.e., all of the physical quantities do not depend on $P_{s}$ and have no correlation to the fluctuation of $P_{s}^{2} / 2 Q$. For this reason, the weight which gives the temperature derivative is not the total energy but the potential energy of the heat bath. 


\section{References}

1) H.C.Andersen: J. Chem. Phys. 72(1980)2384.

2) J.M.Haile, S.Gupta: J. Chem. Phys. 79(1983)3067.

3) S.Nosé: Mol. Phys. 52(1984)255.

4) S.Nosé: J. Chem. Phys. 81(1984)511.

5) W.G.Hoover: Phys. Rev. 31(1984)1695.

6) A.Brańka, M. Parrinello: Mol. Phys. 58(1986)989. 